\title{
Application of Multisim in Digital Logic Design Experiment Teaching
}

\author{
Yuanzi He and Renbo Xu
}

(NanChang Institute of Science and Technology, Nanchang,330108)

Keywords: Multisim simulation technology; digital logic design; experimental teaching

\begin{abstract}
The focus of electronic engineering teaching is to cultivate students' analytical thinking skills so that students can understand how to solve problems independently. At this stage, it is advocated that the simulation technology should be applied to the teaching of digital circuit logic design experiments. This paper has applied research on the experimental design of digital logic design in Multisim, and the experimental results have great guiding significance for the simulation design.

The traditional digital circuit logic design teaching mainly adopts the unilateral education method. Students only rely on the teacher's classroom explanation to complete the task of learning, the passive acceptance of the learning method and the instillation of the learning content make it difficult to achieve the desired educational effect. It is difficult for students to really understand the logic design principles of digital circuits, resulting in poor teaching quality. With the rapid development of computer electronics technology in China, using these softwares for classroom education can make teaching be more intuitive and beneficial to students' understanding and learning.
\end{abstract}

\section{Introduction of Multisim}

The simulation software is a principle capture and interaction simulation software introduced by the US National Instrument Co., Ltd. The software has a rich component library, which contains a large number of digital devices, supporting 51 series single-chip computers and pic single-chip computers, and has a very complete virtual test instrument to support its operation, mainly including universal meters, function signals, generators, oscilloscopes, frequency meters, and so on.

The software has innovative circuit design technology, which can synchronize theoretical teaching with experimental teaching, making the design operation be more systematic and intuitive. At the same time, it also overcomes many obstacles and problems encountered in electronic teaching and has obvious advantages compared with traditional education methods. ${ }^{[1]}$

\section{Application of Multisim in Teaching Process}

At this stage, the development of science and technology in our country is accelerating, and the educational model of electronic engineering has also begun to innovate actively. When teaching the logic design of digital circuits, students should change from easy to difficult, from shallow to deep, so that students can have a process of adapting to the educational model. The following are some applications of Multisim in the teaching process. ${ }^{[2]}$

1 Logic function of simulation gate circuit

The logic function of simulation gate circuit is the basic knowledge of digital logic circuit. Master the logic function and divide it into several steps to carry out teaching, including basic concepts, basic theories and basic methods, which are the keys to carry out learning. The following will show how to simulate the function of xor gate with this software. ${ }^{[3]}$

First of all, turn on the software, establish the working principle diagram, select the power supply and ground wire in the component library, select the single-pole and double-throw switches, set them to "A" and "B", then choose to look at an xor gate, set the voltage to $2.5 \mathrm{~V}$, and operate the system (as shown in Fig. 1). 


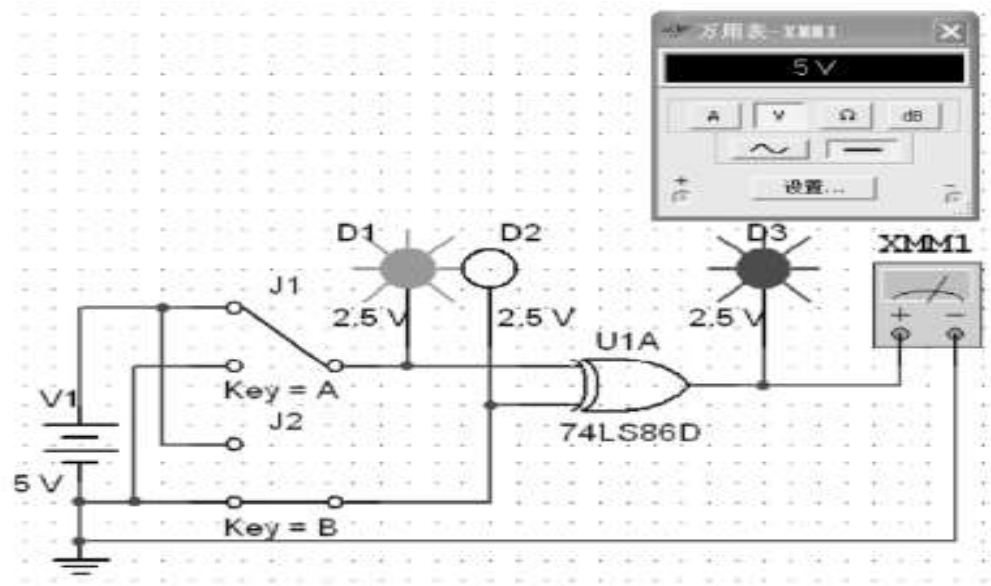

Figure 1. Finite Simulation gate circuit

When the system carries out simulation, through the control of the single-pole double-throw switch, the output and input logic levels can be changed. If lights are on, correspond to the high level and if off, to the low level. Universal meter can intuitively reflect the situation of the output voltage terminal.

2 Application of simulation in combinational logic circuit

After students master the logic function of simulation gate circuit, they can begin to learn simulation combinational logic circuit which is an important part of digital logic and plays a role of connecting the preceding and the following in the digital logic circuit. The knowledge involved in combinational logic circuit is relatively extensive. During learning, students can consolidate algebra to a great extent. Mastering the functions of combinational logic circuits will be of great help to the following study, which will lay a foundation for the overall cognition of electronic engineering. At the same time, it is also an exercise of students' analytical and innovative abilities. This software can enable students to have a comprehensive understanding of the analysis of logic circuit. The following will take the design of a two-input NAND gate three-person voting circuit as an example to analyze its application in combinational logic circuit. ${ }^{[4]}$

As the name implies, if there are 3 persons who vote the circuit, there should be 3 persons who participate in the vote. The signal should be recognized as being passed before it can work, otherwise it will be regarded as transmission failure. The 3 persons can be set to A, B and C at first, and then they can be set logically. The logic when agreeing is set to 1 , and the logic when not agreeing is set to 0 . During the operation of the software, all the output signals should be matched to ensure the integrity of the required signals, and be judged whether to pass or not according to the actual situation of signal transmission so as to obtain the output logic mode (as shown in Fig. 2).

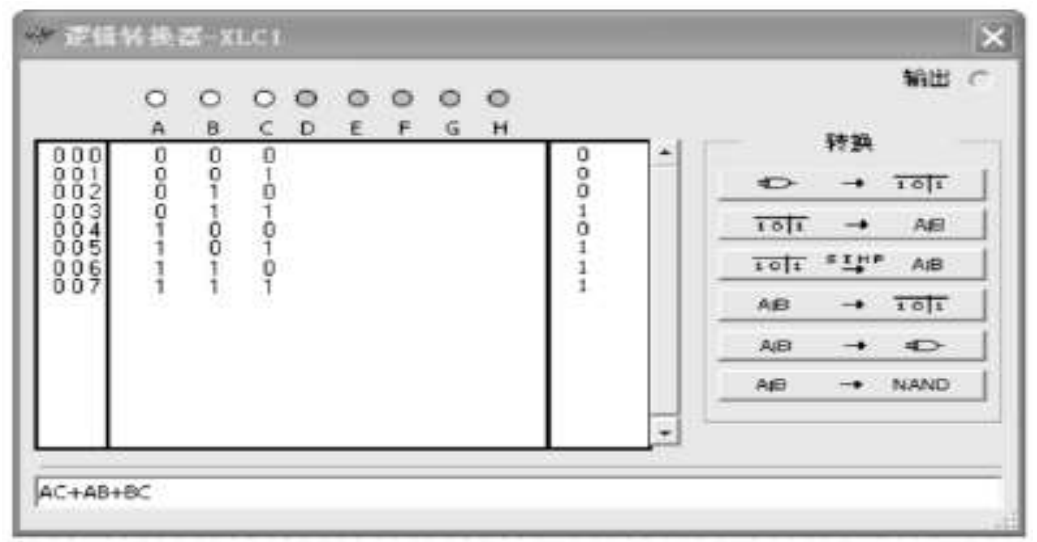

Figure 2. Finite Logic converter

Through examples, it can be seen that this tool software has ideal effect in practical application and can design combinational logic circuit more conveniently. Comparing with the traditional design method of combinational subgrade circuit, it can be found that it saves a large part of 
operating time and also saves operating costs to a certain extent. In addition, this technology can also simulate circuit. Teachers often involve in the design and comparison of various circuit simulations when teaching theories. Using this software to teach can greatly broaden students' eyes, deepen their interests in logic circuit design and improve their understanding abilities. ${ }^{[5]}$

3 Application of simulation in sequential logic circuit

The application in sequential logic circuit is the key point in logic circuit learning, and it is also a difficult part in logic circuit learning process. When students learn about it, it is often not easy to fully understand it. In learning process, they must understand the significance of sequential waveform. Therefore, when explaining this part of contents, teachers should pay attention to use the most intuitive method to make students understand the sequential simulation logic circuit and explain through the intuitive internal signal waveform.

Usually, counter with various structures should be selected. Students should master the design method skillfully and continuously strengthen it. In order to deepen students' understanding of logic circuit, the author uses digital tube to directly display the counter (as shown in Fig. 3) when teaching digital circuit to students, and sends clock pulse and counting output at this time to logic analyzer.

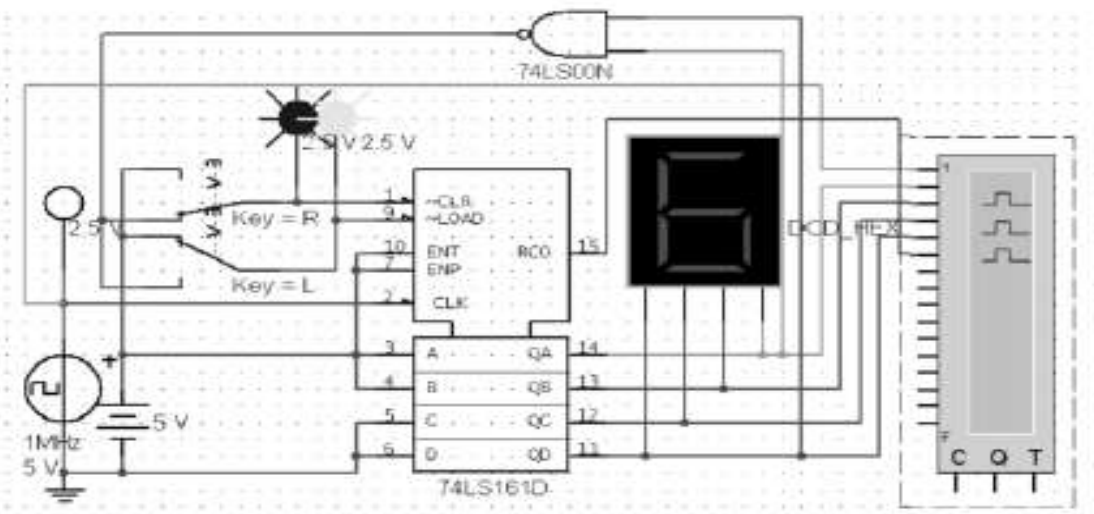

Figure 3. Finite Digital tube

When reaching a high level, attention should be paid to count it accurately to ensure that the output and variation are within the ideal range. When the level position is relatively high, the setting method design can be realized to a certain extent. The relationship between the counting output and the clock pulse can be analyzed by using a logic analyzer.

The use of logic analyzer is closely related to the allocation of channels. Channel 1 should be a clock pulse, and Channel 2 to 5 refer to different sampling frequencies. When the instantaneous state appears on the counter, the reset state should be cleared. Finally, the result of logical analysis is sampled.

\section{Conclusion}

The application of simulation software Multisim in digital circuit logic design is an educational attempt. In the process of teaching, a virtual circuit simulation system should be established on the computer platform, which greatly facilitates the design of various logic circuits, is conducive to students' understanding of the characteristics of the circuits, and can also play a role in guiding students to think independently and innovatively. to a certain extent, which improves students' learning enthusiasm and enables electronic engineering teaching to achieve ideal results. Project funding:Nanchang DME Photoelectric Engineering Key Laboratory (No.NCZDSY -004)

\section{References}

[1] Zhang Yajun, Chen Long, Niu Xiaoyan. Application of Multisim in Digital Circuit and Logic 
Design Experiment Teaching[J]. Experimental Technology and Management, 2014, 20(08): 108-110+114.

[2] Wang Zhe. Multisim in Application of Combinational Logic Circuit Analysis Experiment Teaching[J]. Shandong Industrial Technology, 2017, 28 (02): 292-293.

[3] Ni Deke. Application of Multisim in Digital Circuit Logic Design Teaching[J]. Journal of Xi'an University of Posts and Telecommunications, 2016, 16(S1): 90-92.

[4] Yu Lingyan. Application of Multisim in Digital Logic Circuit[J]. Science, 2014,21(23): $720+661$.

[5] Liang Xianghong. He Baoxiang. Cultivate Students' Abilities in Teaching of "Digital Circuit Logic Design"[J]. China Electric Power Education, 2015, 24(10): 69-70.

[6] Li Yudong. Simple Electronic Piano Design Based on 555 Timer[J]. Science and Technology Wind, 2016 (2): 6. 